\title{
Study on Corrosion Behavior of Copper-Clad Steel for Grounding Grids
}

\author{
Xinhua Zhang *, Zhongkang Zhou, Xiaochun Chen, Jishuang Song and \\ Maolin Shi
}

State Grid Anhui Electric Power Company Electric Power Research Institute, Hefei 230601, China Corresponding author e-mail: 2606030831@qq.com

Keywords: Study, Corrosion Behavior, Copper-Clad Steel.

\begin{abstract}
Grounding grid is an important device guaranteeing the operation of the people, electrical equipments and power grid. However, the corrosion of grounding grid affects the security of the power grid system, what's worse, it makes huge economic losses and serious social impact. We found from the research that the copper-clad steel for grounding grids has any advantages to better economic gain and good corrosion resistance at the same time. In this paper, using electrochemical impedance spectroscopy (EIS), polarization curve measurement, scanning electron microscopy (SEM) and so on, the corrosion behavior of copper-clad steel for grounding grids in the coastal soil have been researched. The results indicated that an increase of the soil salinity decrease the corrosion resistance of copper-clad steel, and the latter stage is less than the mid-term stage. The resistance increase and then tend to be stable with the increase of immersion time. The corrosion resistance of copper-clad steel in the non-salinized and light salinized soil is better.
\end{abstract}

\section{Introduction}

Corrosion of grounding grids in soil is a complex phenomenon, because soil is a heterogeneous system of porous material, with the space between particles filled with water or gases [1]. Yan [2] found that the corrosivity of the red clay soil greatly relieves after Fe oxides are removed from the soil. Victor Padilla [3] studied the effect of de-icing salts on the corrosion performance of galvanized steel in sulphate contaminated soil, and found that the presence of sulphate in the soil significantly increased the corrosion rates. However, such studies about the corrosion behavior of copper-clad steel are less.

In this work, the corrosion behavior of copper-clad steel for grounding grids in the coastal soil was studied by EIS, polarization curve measurement and SEM. The results of this paper may provide an insight on improving the corrosion resistance of copper-clad steel for grounding grids in the coastal soil.

\section{Experimental}

\subsection{Sample and soil solution preparation}

Corrosion experiments were conducted on electrodes made from copper-clad steel, mounted in epoxy resin to give an exposed area of $1.0 \mathrm{~cm}^{2}$ to the media. A copper wire was connected to the backside of each specimen with soldering tin paste. Before each experiment, the working surfaces of the electrodes were polished with different grades of emery paper, and then degreased with ethanol, subsequently cleaned with deionized water, and finally dried in air. In order to avoid a prolonged exposure to the atmosphere, the specimens were tested immediately after the degreasing and drying processes.

The test solution was prepared by adding distilled water and soil from Shanghai China. The extracted soil solution was prepared by mixing soil with distilled water in a ratio of $1: 1$, then adjusted the $\mathrm{Cl}^{-}$content of soil solution by adding $0 \mathrm{~g} / \mathrm{kg}, 2 \mathrm{~g} / \mathrm{kg}, 6 \mathrm{~g} / \mathrm{kg} \mathrm{NaCl}$, which corresponds to nonsalinized soil solution, light salinized soil solution and moderate salinized soil solution. Chemical analysis was carried out to determine the soil chemistry [4] and the results are shown in Table 1. 
Table1. Ionic composition of Shanghai soil, $(\mathrm{g} / \mathrm{kg})$

\begin{tabular}{|l|c|c|c|c|c|c|c|}
$\mathrm{Ca}^{2+}$ & $\mathrm{Mg}^{2+}$ & $\mathrm{K}^{+}$ & $\mathrm{Na}^{+}$ & $\mathrm{SO}_{4}^{2-}$ & $\mathrm{Cl}^{-}$ & $\mathrm{HCO}^{3-}$ & $\mathrm{pH}$ \\
\hline 0.26 & 0.23 & 0.30 & 0.68 & 0.52 & 1.65 & 0.31 & 8
\end{tabular}

\subsection{Electrochemical measurements}

Electrochemical measurements were carried out in a conventional three-electrode cell with a platinum counter electrode and a saturated calomel electrode (SCE) as reference. The EIS measurements were carried out at open circuit potential over a frequency range from $0.05 \mathrm{~Hz}$ to 100 $\mathrm{kHz}$ with sinusoidal potential perturbation $5 \mathrm{mV}$ by using 2273 type electrochemical workstation. The impedance data were analyzed with the ZSIMPWIN software and fitted to the appropriate equivalent circuits. The polarization curves were performed at a potential scan rate of $5 \mathrm{mV} \cdot \mathrm{s}^{-1}$. The potential range was from -0.35 to $0.15 \mathrm{~V}$. All the potential values in this paper were relative to the saturated calomel electrode (SCE).

\subsection{Characterization of corrosion products}

The micro-structure morphologies of the copper-clad steel were analyzed using the Hitachi scanning electron microscope (SU-1500, all-in-one machine).

\section{Results and discussion}

\subsection{Electrochemical impedance spectroscopy}

The Nyquist impedance plots of copper-clad steel electrodes immersed in different salinity in soil solution are shown in Fig 1. The impedance data of copper-clad steel at immersion times for $1 \mathrm{~d}, 91$ $\mathrm{d}$ and $160 \mathrm{~d}$ represents the early, medium and late. Two equivalent electrical circuits were given in Fig 2 and were employed to fit the EIS of the copper-clad steel electrodes immersed in different salinity in soil solution. In the two equivalent circuit models, $\mathrm{Rs}$ is the soil solution resistance, $\mathrm{R}_{1}$ and $\mathrm{C}_{1}$ corresponds to the resistance and capacitance of the surface film of rust layer, $\mathrm{R}_{\mathrm{ct}}$ and $\mathrm{C}_{2}$ is the charge transfer resistance and the double-layer capacitance.

Fig. 3 showed the time dependence of $R_{1}$ and $R_{c t}$ of copper-clad steel in different salinity soil solution from the curve fitting data. Apparently, the $\mathrm{R}_{1}$ quickly increased to the maximum values with time, then reduced and tend to be stable. The $R_{c t}$ increased and then kept invariant. The larger an $R_{c t}$ value, the greater the reaction resistance, indicating that the corrosion resistant of copper-clad steel increased with time. By comparing the corrosion rate at the same time in the different salinity soil solution, $\mathrm{R}_{\mathrm{ct}}$ in the light salinized soil solution was lower than that in other soil medium, but not that far away, indicating that the corrosion resistant of copper-clad steel decreased with the soil salinity increase.
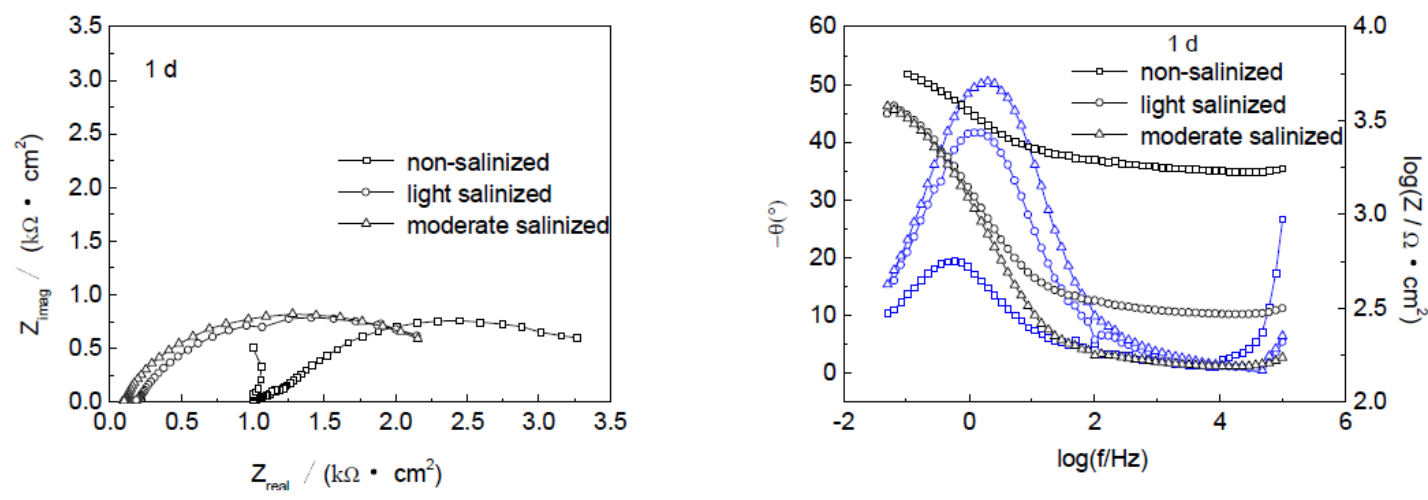

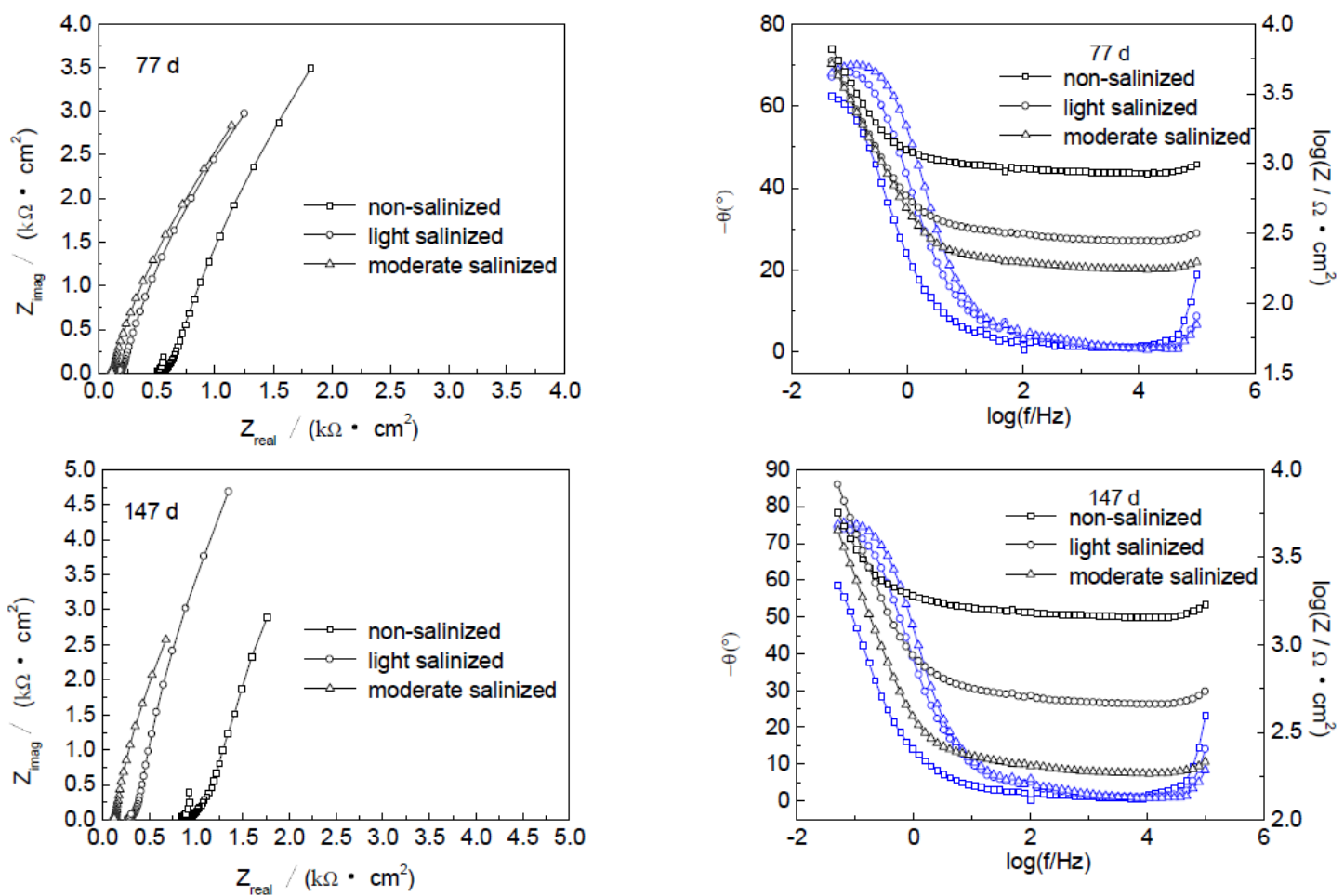

Fig 1. Nyquist and Bode plots of copper-clad steel in different salinity soil solution

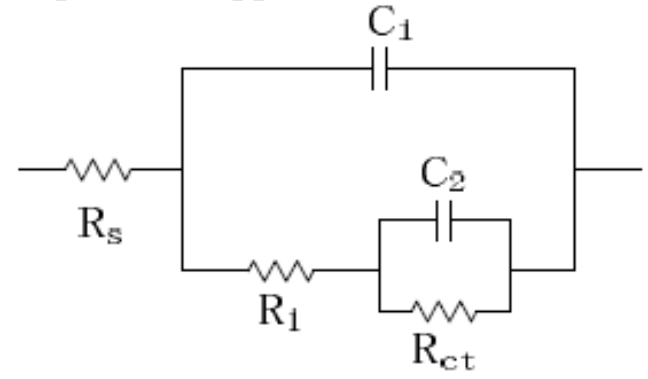

Fig 2. Equivalent circuit models used to fit the experiment impedance data
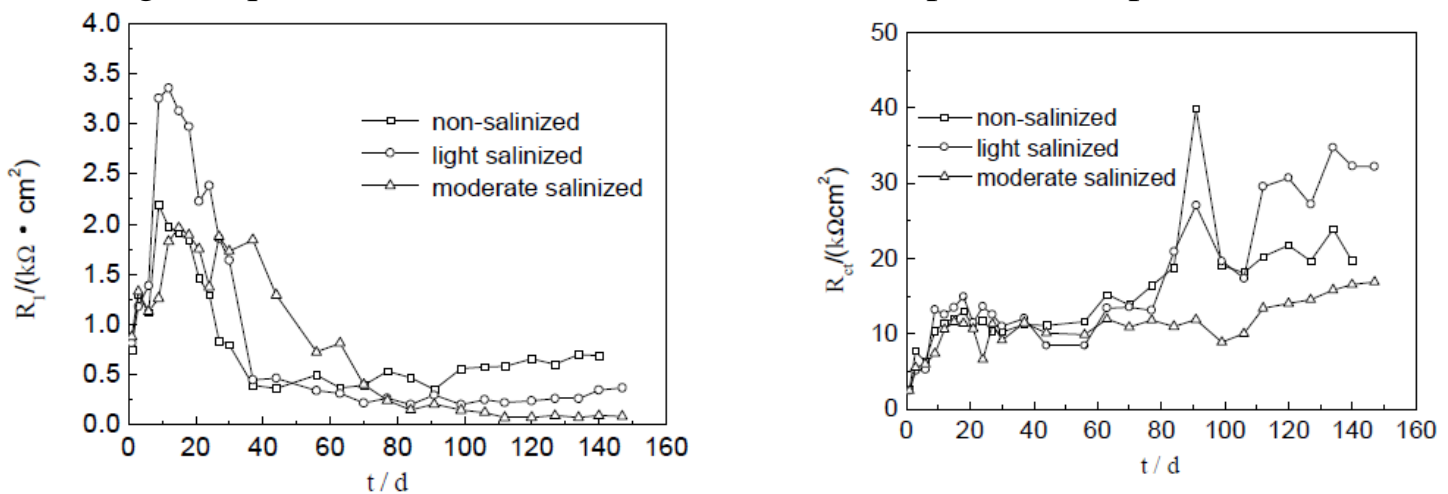

Fig 3. The change of R1 and Rct on copper-clad steel in different salinity soil solution

\subsection{Polarization curve measurements}

Typical polarization curves recorded for the copper-clad steel after $92 \mathrm{~d}$ and $160 \mathrm{~d}$ in different salinity soil solution are shown in Fig. 4. It is shown that the passivation region did not exist in anodic region of copper-clad steel, indicating that the passivation of copper-clad steel did not take place in different salinity soil solution. The copper-clad steel corrosion process in soil was under the activation (electrochemical) control. As the salinity increased, the slope of anodic polarization curves changed very little, indicating that the soil salinity did not cause the changes of anode reaction mechanism of copper-clad steel.

The corrosion potentials $\left(\mathrm{E}_{\text {corr }}\right)$ and the corrosion current density $\left(\mathrm{I}_{\mathrm{corr}}\right)$ were listed in Table 2 . $\mathrm{I}_{\text {corr }}$ in the light salinized soil solution was larger than that in the other soil at the same time. With 
increasing salinity of the soil solution, the corrosion current density of copper-clad steel increased for 92 days. For 160 days, the corrosion current density of copper-clad steel in non-salinized soil solution comparable to that in light salinized soil solution. The Icorr for 160 days increased and then tend to be stable with increase of soil salinity, but less than the corrosion rate for 92 days. According to the corrosion level standard [5], the corrosion degree of copper-clad steel electrode corrosion which buried after 160 days are the second level $\left(3<\right.$ Icorr $\left.<10 \mu \mathrm{A} \times \mathrm{cm}^{-2}\right)$, that is, medium corrosion.
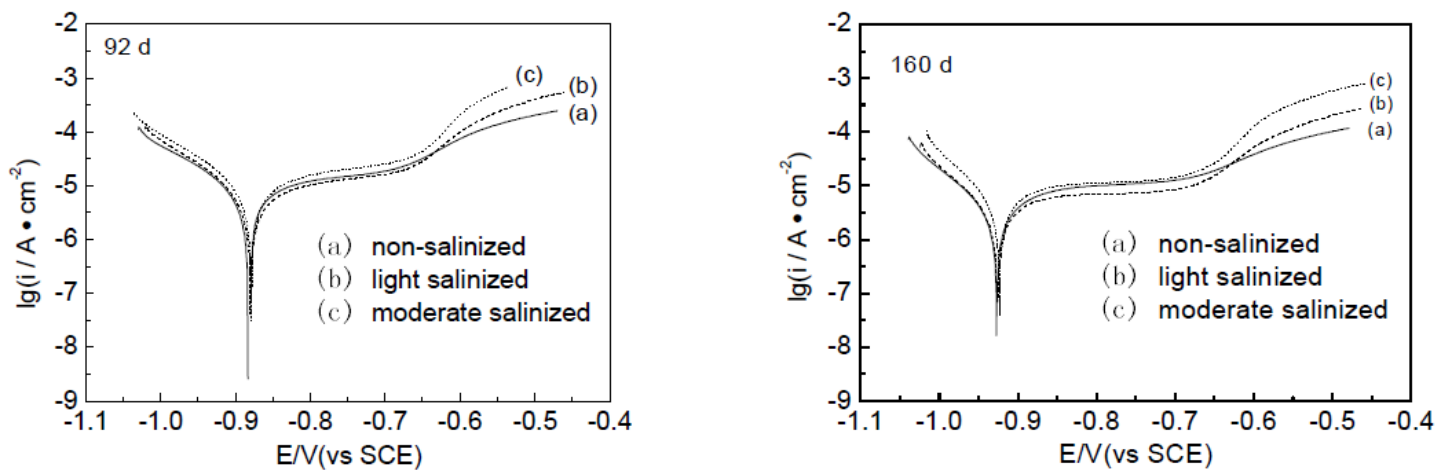

Fig 4. The polarization curves of copper-clad steel in different salinity soil solution

Table 2. Results of Tafel curves for copper-clad steel in different salinity soil solution

\begin{tabular}{|c|c|c|c|c|}
\hline \multirow{2}{*}{ Salinity } & \multicolumn{3}{|c|}{$92 \mathrm{~d}$} & \multicolumn{2}{c|}{$160 \mathrm{~d}$} \\
\cline { 2 - 5 } & $\mathrm{E}_{\text {corr }} / \mathrm{mV}$ & $\mathrm{I}_{\text {corr }} /\left(\mu \mathrm{A} \times \mathrm{cm}^{-2}\right)$ & $\mathrm{E}_{\text {corr }} / \mathrm{mV}$ & $\mathrm{I}_{\text {corr }} /\left(\mu \mathrm{A} \times \mathrm{cm}^{-2}\right)$ \\
\hline non-salinized & -882.629 & 7.796 & -926.872 & 4.428 \\
\hline light salinized & -879.615 & 9.078 & -925.660 & 4.211 \\
\hline moderate salinized & -879.616 & 11.480 & -923.103 & 6.815 \\
\hline
\end{tabular}

\subsection{Surface characterization}

Copper-clad steel electrodes from in different salinity soil solution were removed immediately, then cleaned with deionized water, and steel layers surface of copper-clad steel electrode were greygreen, subsequently changed to reddish-brown. Fig.5 showed the SEM images of the copper-clad steel surface. These images presented the copper-clad steel surface had a different degree of corrosion, the combination of the two materials in moderate salinized soil solution showed obvious cracks.

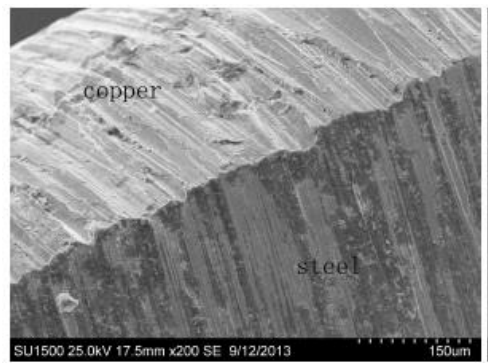

(a) non-salinized

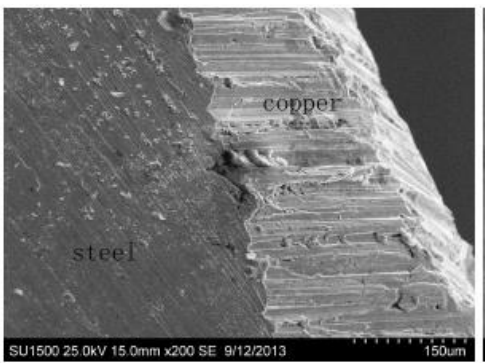

(b) light salinized

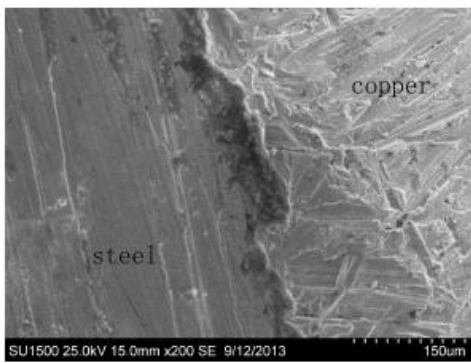

(c) moderate salinized

Fig5. The SEM images for copper-clad steel in different salinity soil solution

\section{Conclusion}

Copper-clad steel in non-salinized and light salinized soil solution showed good resistance to corrosion, the corrosion degree is medium corrosion. With increasing salinity of the soil solution, the corrosion resistant of copper-clad steel decreased. With the buried duration, the resistance increase and then tend to be stable, the corrosion resistant of copper-clad steel increased with time.

The corrosion of copper-clad steel in moderate soil solution was more serious. In moderate salinized soil, the copper clad steel had the lowest impedance value, the corrosion current density reached to maximum, and the combination of the two materials showed obvious cracks. 


\section{References}

[1] Vanessa de Freitas Cunha Lins, Mitchel Leonard Magalhães Ferreira, Patrícia Alves Saliba. Corrosion Resistance of API X52 Carbon Steel in Soil Environment [J]. Journal of Materials Research and Technology, 2012, 1(3): 161-166.

[2] Maocheng Yan, Cheng Sun, Jin Xu, et al. Role of Fe oxides in corrosion of pipeline steel in a red clay soil [J]. Corrosion Science, 2014, 80: 309-317.

[3] Victor Padilla, Pouria Ghods, Akram Alfantazi. Effect of de-icing salts on the corrosion performance of galvanized steel in sulphate contaminated soil [J]. Construction and Building Materials, 2013, 40: 908-918.

[4] Yang Dong, Ruijun Hao, Hailan Fang,et al.Analysis of the Soil Salinity in Shanghai Lingang Heavy-Facility Area [J]. Journal of Shanghai Jiaotong University (Agricultural Science), 2008, 26(8): 578-583.

[5] Hongyan Tang, Guangling Song, Chunan CAO, et al. Corrosion Behavior of carbon Steel in Soil by Polarization Curves [J]. Corrosion Science and Protection Technology, 1995, 7(4): 285 - 292. 